\title{
Test of ATLAS Micromegas detectors with a ternary gas mixture at the CERN GIF++ facility
}

\author{
Fabian Vogel on behalf of the ATLAS Muon Collaboration ${ }^{a, *}$ \\ ${ }^{a}$ LMU München, \\ Am Coulombwall 1, Garching, Deutschland \\ E-mail: fabian.vogel@physik. uni-muenchen.de
}

The ATLAS collaboration at LHC has chosen the resistive Micromegas technology, along with the small-strip Thin Gap Chambers (sTGC), for the high luminosity upgrade of the first muon station in the high-rapidity region, the New Small Wheel (NSW) project. Four different types of Micromegas quadruplets have been constructed at four construction sites in Italy (SM1), Germany (SM2), France (LM1) and CERN/Greece/Russia (LM2). At CERN, the final validation and the integration of the modules into Sectors and their commissioning are in progress. The achievement of the requirements for these detectors revealed to be even more challenging than expected. One of the main features being studied is the HV stability of the detectors. Several approaches have been tested in order to enhance the stability, among them the use of different gas mixtures. A ternary Argon- $\mathrm{CO}_{2}-\mathrm{iC}_{4} \mathrm{H}_{10}$ mixture has shown to be effective in dumping discharges and dark currents. It allows the operation of the Micromegas detectors at working points with high cosmic muon detection efficiency. The presence of Isobutane in the mixture required a set of aging studies, ongoing at the GIF++ radiation facility at CERN, where the expected HL LHC background rate is reached by a ${ }^{137} \mathrm{Cs} 14 \mathrm{TBq}$ source of $662 \mathrm{keV}$ photons. Preliminary aging results and effectiveness of the ternary mixture will be shown.

\footnotetext{
*** The European Physical Society Conference on High Energy Physics (EPS-HEP2021), ***

*** 26-30 July $2021 * * *$

*** Online conference, jointly organized by Universität Hamburg and the research center DESY ***
}

\footnotetext{
${ }^{*}$ Speaker
} 


\section{Performance studies of the ATLAS New Small Wheel Micromegas}

The ATLAS [1] New Small Wheels are built from $2 \mathrm{~m}^{2}$ (SM) and $3 \mathrm{~m}^{2}$ (LM) resistive strip Micromegas detectors. One challenging aspect is the High Voltage (HV) stability of the large area resistive strip Micromegas detectors. Besides enlarging the quenching resistance by passivating active area at the detector edges, studies on different gas mixtures are ongoing in GIF++ (CERN) and at the LMU Munich. A ternary gas mixture containing Argon (Ar), Carbon dioxide $\left(\mathrm{CO}_{2}\right)$ and Isobutane $\left(\mathrm{iC}_{4} \mathrm{H}_{10}\right)$ with a ratio of $93: 5: 2 \mathrm{vol} \%$ is investigated as a viable replacement of the standard gas mixture of $\mathrm{Ar}: \mathrm{CO}_{2} 93: 7$ vol\%.

\section{Long Term irradiation at the CERN Gamma Irradiation Facility GIF++}

The $662 \mathrm{keV}$ gamma irradiation at the GIF++ is produced by a $14 \mathrm{TBq}{ }^{137} \mathrm{Cs}$ source. This source is capable to simulate the irradiation intensity expected for the High-Luminosity upgrade of the LHC (HL-LHC, [2]). First studies were performed with series production Micromegas from the four production sites and foreseen for integration in NSW-C.

Figure 1 shows the position of the gamma source as well as the position of the five irradiated Micromegas detectors. SM1 M35 is a reference chamber running with the standard gas mixture of Ar: $\mathrm{CO}_{2}=93: 7 \mathrm{vol} \%$. SM1 M31, LM1 M07 and LM2 M20 were operated successfully for 4-5 months with $\mathrm{Ar}: \mathrm{CO}_{2}: \mathrm{iC}_{4} \mathrm{H}_{10}=93: 5: 2$ vol\% at a distance of $3 \mathrm{~m}$ from the source. At this position the interaction effect is about the same as the maximum particle rate expected at the NSW detectors during the HL-LHC phase [3]. SM2 M33 is positioned closer to the source at $1 \mathrm{~m}$ distance increasing the interaction rate by a factor of 8 . This chamber was tested for about 2 weeks and showed extremely stable performance and almost constant gain even at highest irradiation intensities.

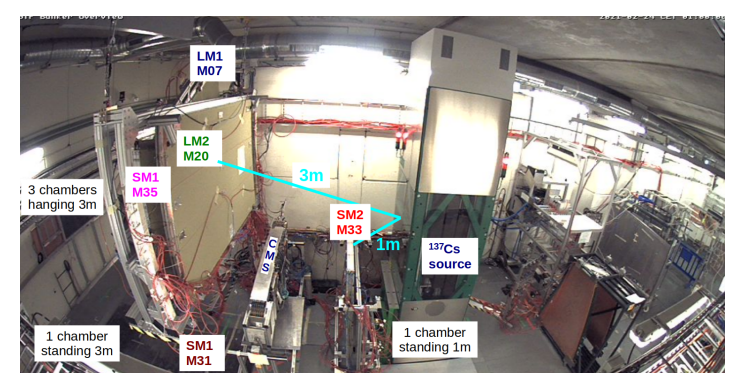

Figure 1: Picture of the experimental setup in the GIF++. Three Micromegas modules are placed in a hanging trolley at $3 \mathrm{~m}$ distance from the source. SM1 M31 stands below this trolley. One module (SM2 M33) is positioned at $1 \mathrm{~m}$ distance from the source.

\section{Efficiency comparison for cosmic muon tracking beore and after irradiation}

Tracking efficiencies of the NSW modules are determined by position reconstructions of cosmic muon tracks inside of the detector layers. The reconstructed position is requested to be within $5 \mathrm{~mm}$ or $10 \mathrm{~mm}$ distance to a known reference track for eta and stereo layers respectively. A comparison of this efficiency of one double wedge (DW) of the NSW for the two different gas mixtures is shown in figure 2. Detectors filled with the ternary gas mixture are operated at voltages up to $520 \mathrm{~V}$ where they exceed the efficiency of the detectors operated with the standard gas mixture at the working point of $570 \mathrm{~V}$. 


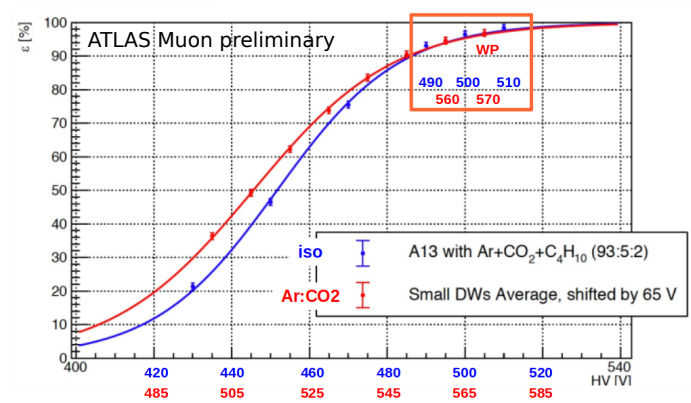

Figure 2: Muon tracking efficiency of one DW of the NSW. The red line shows the efficiency using the ternary gas. The efficiency for the standard gas mixture is represented by the blue line that is shifted by $65 \mathrm{~V}$ for direct comparison of the efficiency curves. Higher efficiencies at comparable voltages are achieved with the admixture of Isobutane.

\section{HV stability improvement of a NSW Double Wedge}

A comparison of the radiation induced current for one layer of such a Micromegas detector for the two gases shows the positive effect of the admixture of Isobutane (see fig. 3, bottom row). The five discharging sectors at $570 \mathrm{~V}$ (Ar: $\mathrm{CO}_{2}$, see fig. 3, upper row) do not longer discharge at $520 \mathrm{~V}$ (Ar: $\mathrm{CO}_{2}: \mathrm{iC}_{4} \mathrm{H}_{10}$ ).

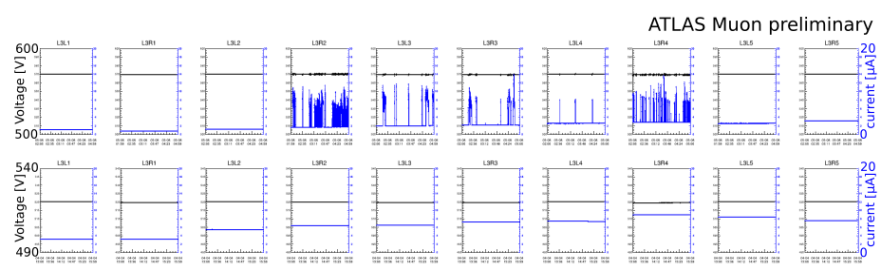

Figure 3: Voltages (black) and currents (blue) over three hours for a single Micromegas layer under $\mathrm{Ar}: \mathrm{CO}_{2}$ (top) and $\mathrm{Ar}: \mathrm{CO}_{2}: \mathrm{iC}_{4} \mathrm{H}_{10}$ (bottom) in the $\mathrm{GIF}++$. The addition of Isobutane reduces the discharge intensity to zero, i.e. no more blue current spikes.
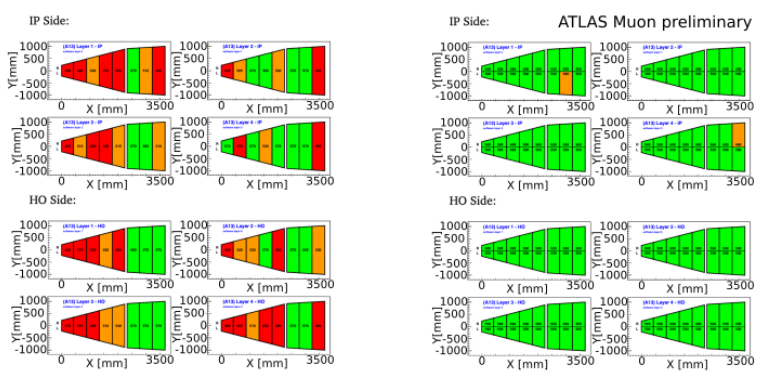

Figure 4: Reachable HV levels for a complete DW of the NSW for standard gas (left) and the ternary gas mixture (right). Red and orange sectors represent voltages, that correspond to expected efficiencies $<90 \%$. By adding Isobutane to the gas mixture all but two sectors surpass this crucial limit, compared to the standard gas, where many sectors do not reach it. The Micromegas detectors were not yet passivated during this measurement. Meanwhile the detectors are passivated with improved performance under $\mathrm{Ar}: \mathrm{CO}_{2}$. 
Figure 4 emphasizes clearly the improvement by adding Isobutane. The left figure shows an overview over all HV sectors of one DW under standard gas. Many sectors (red and orange) do not reach the desired voltages $(>550 \mathrm{~V})$. The same DW reaches $490 \mathrm{~V}$, i.e. equal efficiencies are achieved (see fig. 2) for all but two sectors under Isobutane (see fig. 4, right).

\section{Neutron irradiation of an ATLAS NSW Micromegas module}

Additional irradiation tests with neutrons from an $3.7 \mathrm{GBq}$ Americium-Beryllium source $\left(<\mathrm{E}_{\mathrm{n}}>=4-5 \mathrm{MeV}\right)$ performed in the MLL showed compatible gains for $570 \mathrm{~V}$ under $\mathrm{Ar}: \mathrm{CO}_{2}$ and $515 \mathrm{~V}$ under $\mathrm{Ar}: \mathrm{CO}_{2}: \mathrm{iC}_{4} \mathrm{H}_{10}$ (see fig. 5 left and right).
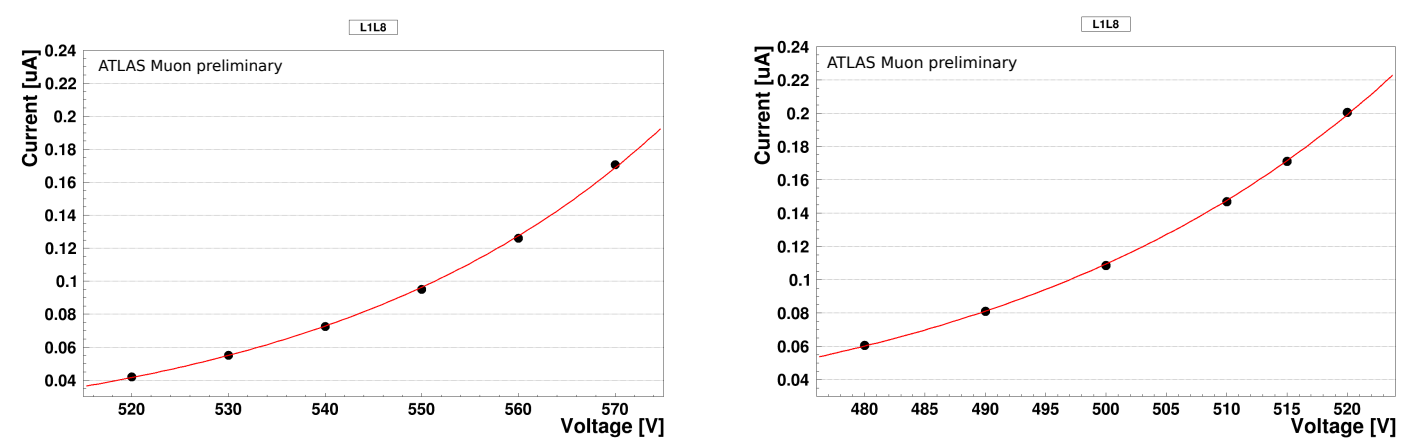

Figure 5: Ramp-up scans under neutron irradiation. Shown are the curves for the highest irradiated sector under Ar: $\mathrm{CO}_{2}$ (left) and $\mathrm{Ar}: \mathrm{CO}_{2}: \mathrm{iC}_{4} \mathrm{H}_{10}$ (right). Comparable gains are reached for $570 \mathrm{~V}$ under standard gas and at $515 \mathrm{~V}$ under the ternary gas mixture. After 1.5 years of irradiation no decrease in gain was observed.

\section{Conclusion}

By addition of Isobutane to the standard operating gas mixture of the NSW Micromegas detectors a better High Voltage stability is achieved using the teranry gas mixture at a considerably lower operating voltage. Despite the lower voltages higher tracking efficiencies are obtained. Additionally sectors operating under $\mathrm{Ar}: \mathrm{CO}_{2}: \mathrm{iC}_{4} \mathrm{H}_{10}$ discharge less. The aging tests will continue to reach an equivalent dose to 10 years at HL-HLC. 1.5 years long neutron irradiation tests at LMU Munich have shown similar gains for detectors under $\mathrm{Ar}: \mathrm{CO}_{2}: \mathrm{iC}_{4} \mathrm{H}_{10}$ at $515 \mathrm{~V}$ and under $\mathrm{Ar}: \mathrm{CO}_{2}$ at $570 \mathrm{~V}$. The SM2 Micromegas chamber was running without any problems the whole period.

\section{References}

[1] ATLAS Collaboration, The ATLAS Experiment at the CERN Large Hadron Collider, JINST 3 S08003 (2008)

[2] Guida, Roberto GIF++: The new CERN Irradiation Facility to Test Large-Area Detectors for the HL-LHC Program, PoS ICHEP2016 260, (2016)

[3] Dorothea Pfeiffer, et. al The Radiation Field in the New Gamma Irradiation Facility (GIF++) at CERN, arXiv:1611.00299v1 [physics.ins-det], (2016) 